\title{
In vitro and in vivo effects of short hairpin RNA targeting integrin-linked kinase in prostate cancer cells
}

\author{
YEQING YUAN $^{1 *}$, YUNBEI XIAO ${ }^{2 *}$, QING LI ${ }^{1}$, ZHENHUA LIU $^{1}$, XIAOWEI ZHANG ${ }^{1}$, \\ CAIPENG QIN ${ }^{1}$, JING XIE $^{1}$, XIAOFENG WANG ${ }^{1}$ and TAO XU ${ }^{1}$ \\ ${ }^{1}$ Department of Urology, Peking University People's Hospital, Beijing 100044; ${ }^{2}$ Department of Urology, \\ Wenzhou People's Hospital, Wenzhou 325000, P.R. China
}

Received February 9, 2013; Accepted June 14, 2013

DOI: $10.3892 / \mathrm{mmr} .2013 .1532$

\begin{abstract}
Integrin-linked kinase (ILK) localizes at focal adhesion sites, plays an important role in cell-matrix interactions and is involved in the regulation of tumor cell growth and migration. The aim of the present study was to clarify the functional characterization of ILK in prostate cancer (PCa) cells. ILK was shown to be overexpressed in 57.1\% (36/63) of PCa samples and $18.2 \%(2 / 11)$ of benign prostatic hyperplasia (BPH) samples using immunohistochemical analysis. DU145 PCa cells knocked down for ILK were examined using western blot analysis, proliferation assay, flow cytometry and wound healing assay. Depletion of ILK significantly impaired cell growth and motility, induced apoptosis in vitro, and delayed xenograft tumor proliferation in nude mice, which were important for oncogenesis and tumor progression. Western blot analysis showed that Akt activity was attenuated in ILK-depleted cells compared with the control cells. These results indicate that ILK knockdown attenuates the biological behavior of PCa cells by decreasing Akt activity, demonstrating that ILK is involved in the development and progression of PCa. Thus, ILK is suggested to serve as a potential therapeutic target for PCa.
\end{abstract}

\section{Introduction}

Prostate cancer (PCa) is the most common malignancy and the second leading cause of cancer-related mortality among males in the United States (1). The recommended treatment for patients with progressive carcinoma of the prostate is hormone ablation achieved by surgical or pharmacological castration (2). Most patients initially respond well to this treatment; however, they inevitably relapse and develop an incurable castration-resistant

Correspondence to: Professor Tao Xu, Department of Urology, Peking University People's Hospital, 11 Xizhimen South Street, Beijing 100044, P.R. China

E-mail:xutao@medmail.com.cn

*Contributed equally

Key words: prostate cancer, integrin-linked kinase, gene knockdown, short hairpin RNA condition within a few years (3).PCa involves highly complicated and heterogeneous tumors resulting from the aberrant activation of several important signaling pathways. Understanding the molecular mechanisms underlying PCa is of utmost importance in the investigation of novel therapeutic strategies.

The ILK gene is located on human chromosome 11 (4), encoding a protein which is composed of three major domains: an N-terminal ankyrin repeat domain, a middle pleckstrin homology (PH) domain and a C-terminal kinase domain (5). This gene is expressed as a serine/threonine (Ser/Thr) protein kinase, a binding partner of $\beta 1$ and $\beta 3$ integrin subunit, which is a cytoplasmic effector of integrin receptors, and constitutes a functional link between them and the actin cytoskeleton (6). ILK expression and its oncogenic potential have been studied in various malignancies including ovarian, colon, hepatocellular, laryngeal and bladder cancer (7-13). Previous studies have shown higher ILK expression levels in primary PCa cells compared with adjacent benign prostatic hyperplasia (BPH) cells (14). However, the functional characterization of ILK in $\mathrm{PCa}$ and its potential in vivo downstream effectors still remain to be elucidated.

The aim of the present study was to clarify the functional characterization of ILK in PCa. Therefore, ILK-mediated PCa tumorigenesis was investigated through the determination of ILK expression in human PCa tumor samples, and its role in vitro and in vivo was evaluated.

\section{Materials and methods}

Chemicals, reagents and plasmid. Tissue microarray, containing $63 \mathrm{PCa}$ and $11 \mathrm{BPH}$ samples, was obtained from Ailina Biotechnology Co., Ltd. (Xian, Shanxi, China). Antibodies against ILK and phosphorylated Akt (Ser473) were obtained from Cell Signaling Technology, Inc. (Beverly, MA, USA). RPMI-1640 medium, fetal bovine serum (FBS) and other reagents were obtained from CWBIO Co., Ltd. (Beijing, China). ILK short hairpin RNA (shRNA) was obtained from OriGene Technologies (Beijing, China). The shRNA vector was cloned into a retroviral silencing plasmid (pRS) under a U6 promoter for mammalian cell expression. The disturbance sequence (sense, 5'-uggacaccgugauauugua-3'dTdT and antisense, 5'-accuguggcacuauaacau-3'dTdT) was designed and synthesized according to the GenBank database. 
Immunohistochemical analysis. Immunohistochemistry was performed using the EnVision ${ }^{\mathrm{TM}} \mathrm{ABC}$ kit according to the manufacturer's instructions. The concentration of primary antibody against ILK was 1:100. After counterstaining with hematoxylin, the sections were dehydrated and mounted. A previously established immunohistochemistry scoring system for prostate carcinomas was used as a reference (15). The percentage of positively stained cells was scored as $1,<25 \%$; 2, 25-50\%; and 3,>50\%. The staining intensity was also scored as follows: 0 , absence of signal; 1 , low-intensity signal (light brown); 2, moderate intensity (brown); and 3, high-intensity signal (dark brown). The frequency and intensity scores were added to obtain the final score for each case. The overall results were assigned a negative (-) score when the sum was $<4$, and a positive (+) score when the sum was $\geq 4$.

Cell culture and transfection. DU145 human prostate cancer cells were obtained from the Department of Pathology of Peking University (Beijing, China) and maintained in RPMI-1640 medium supplemented with 10\% FBS, $100 \mathrm{U} / \mathrm{ml}$ penicillin and $100 \mu \mathrm{g} / \mathrm{ml}$ streptomycin. The cells were cultured in a $5 \% \mathrm{CO}_{2}$ incubator (MCO-17AI; Sanyo Electric Co., Ltd., Osaka, Japan) at $37^{\circ} \mathrm{C}$ in a humidified atmosphere and subcultured every 2-3 days. DU145 cells were seeded at a density of 10,000 cells/well in 96-well plates, and cultured in RPMI-1640 medium containing $10 \%$ FBS to $90 \%$ confluence. The medium was replaced with serum/antibiotic-free RPMI-1640 medium prior to transfection. Transfection was performed using Lipofectamine ${ }^{\mathrm{TM}} 2000$ reagent according to the manufacturer's instructions. The final shRNA transfection concentration was $100 \mathrm{nM}$. Following transfection, DU145 cells were allowed to recover in RPMI-1640 medium containing 10\% FBS for $24 \mathrm{~h}$ prior to further examination. The study was approved by the ethics committee of Peking University Peoples' Hospital, Beijing City, China

Protein extraction and western blot analysis. Total cellular protein $(30 \mu \mathrm{g})$ was fractionated on sodium dodecyl sulfate-polyacrylamide gel (SDS-PAGE) and transferred onto nitrocellulose membranes (Amersham Pharmacia Biotech, Inc., Piscataway, NJ, USA). The blots were blocked and incubated with the indicated specific primary antibody. After incubation with the secondary antibody, the signal was visualized using the ECL Western Blot kit and exposed to film (Kodak, Rochester, NY, USA). $\beta$-actin was used as an internal control by incubating a specific antibody against $\beta$-actin (Sigma, St. Louis, MO, USA).

Proliferation assay. Cell viability was assessed using the MTT assay. Briefly, transfected DU145 cells were plated at a density of $1 \times 10^{3}$ cells $/ \mathrm{ml}$ in 96 -well plates. After $48 \mathrm{~h}, 10 \mu \mathrm{l} \mathrm{MTT}$ was added to each well. After $4 \mathrm{~h}$ of incubation at $37^{\circ} \mathrm{C}$, the solution was discarded and the produced formazan was solubilized in $100 \mu \mathrm{l}$ of dimethyl sulfoxide (DMSO). Absorbance was measured at $570 \mathrm{~nm}$ using an automated microplate reader (Bio-Rad 550; Bio-Rad, Hercules, CA, USA). All the experiments were performed in triplicate.

Flow cytometric analysis of apoptosis. The collected cells were washed twice with ice-cold phosphate-buffered saline
(PBS) and centrifuged at $150 \mathrm{x} \mathrm{g}$ for $5 \mathrm{~min}$. Supernatants were discarded and the cells were resuspended in $300 \mu \mathrm{l}$ binding buffer. Five microliters of FITC-conjugated Annexin V (10 mg/ml) and $10 \mu \mathrm{l}$ of propidium iodide (PI; $50 \mathrm{mg} / \mathrm{ml}$ ) were added and the mixtures were incubated for $15 \mathrm{~min}$ at room temperature in the dark. The samples were then immediately analyzed using fluorescence-activated cell sorting (FACS). The cells $\left(1 \times 10^{4}\right)$ were collected and the percentage of apoptotic cells was recorded.

Wound healing assay. Cell mobility was assessed using a scratch wound assay. Transfected cells were cultured in a 6 -well plate until confluent. The cell layer was carefully wounded using sterile tips and washed twice with fresh medium. Following incubation for 0,12 and $24 \mathrm{~h}$, the cells were imaged at low magnification $(\mathrm{x} 40)$. The experiments were performed in triplicate.

Xenograft assay in nude mice. To confirm the effect of downregulated ILK on tumorigenicity in vivo, ILK shRNAor vector-transfected DU145 cells were resuspended in PBS (pH 7.4), mixed with $1 \mathrm{X}$ Matrigel (BD Biosciences, Palo Alto, CA, USA), and subcutaneously injected (5x10\%/injection) into the left flanks of male athymic BALB/c nu/nu nude mice (4-6 weeks old; purchased from Vital River Laboratories, Ltd., Beijing, China). The mice were sacrificed 4 weeks after the injection. Tumor volume was calculated using the following formula: Volume $=$ width $^{2} \mathrm{x}$ length $\mathrm{x}$ 0.5236. Animal-based experiments were approved by the Peking University Animal Care and Use Committee (Beijing, China) and performed in accordance with accepted standards of humane animal care.

Statistical analysis. All statistical analysis was performed using the Statistical Package of the Social Sciences (SPSS) software version 16.0. Data are shown as the mean \pm SEM. Student's t-test was used to determine statistical differences and $\mathrm{P}<0.05$ was considered to indicate a statistically significant difference.

\section{Results}

ILK expression in PCa and BPH tissues. A total of 74 tissue samples were analyzed. ILK expression was detected in the cytoplasm of PCa cells (Fig. 1). Similar to the results of a previous study (14), ILK was expressed in $57.1 \%(36 / 63)$ of the PCa samples and $18.2 \%(2 / 11)$ of the BPH samples, indicating that ILK expression levels were significantly higher in tumor tissues compared with BPH tissues ( $\mathrm{P}=0.017$; Table I). However, in contrast to previously reported results (14), ILK expression was not correlated with the tumor grade of $\mathrm{PCa}$, although there was a moderate increasing percentage among the tissues with a different Gleason score (Table I).

Effects of ILK knockdown on PCa cell growth, migration and apoptosis. An effective way of elucidating the physiological role of ILK in PCa cells is to inhibit the endogenous expression of ILK. Efficient knockdown of ILK expression was achieved by the successful delivery and expression of shRNA targeting ILK into cells. Forty-eight hours after transfection with ILK shRNA, ILK expression was significantly 
Table I. Expression of ILK in PCa and BPH tissues.

\begin{tabular}{|c|c|c|c|c|c|}
\hline Cell type & Cases, $\mathrm{n}$ & Negative staining, n (\%) & Positive staining, $\mathrm{n}(\%)$ & $\chi^{2}$ & P-value \\
\hline $\mathrm{BPH}$ & 11 & $9(81.8)$ & $2(18.2)$ & 5.6904 & 0.017 \\
\hline $\mathrm{PCa}$ & 63 & $27(42.9)$ & $36(57.1)$ & & \\
\hline \multicolumn{6}{|c|}{ Gleason score } \\
\hline $2-4$ & 17 & $8(47.1)$ & $9(52.9)$ & 0.7453 & 0.689 \\
\hline $5-7$ & 31 & $14(45.2)$ & $17(54.8)$ & & \\
\hline $8-10$ & 15 & $5(33.3)$ & $10(66.7)$ & & \\
\hline
\end{tabular}

A total of $63 \mathrm{PCa}$ specimens were analyzed; $57.1 \%$ of the PCa specimens examined were positively stained for ILK, while ILK expression in most of the BPH tissues (9/11) was either absent or weak. The difference between PCa and BPH tissues was significant $(\mathrm{P}=0.017)$. The rate of positively stained PCa tissues moderately increased with inceasing Gleason score (52.9, 54.8 and 66.7\%, respectively), but no statistical significance was observed. ILK, integrin-linked kinase; BPH, benign prostatic hyperplasia; PCa, prostate cancer.
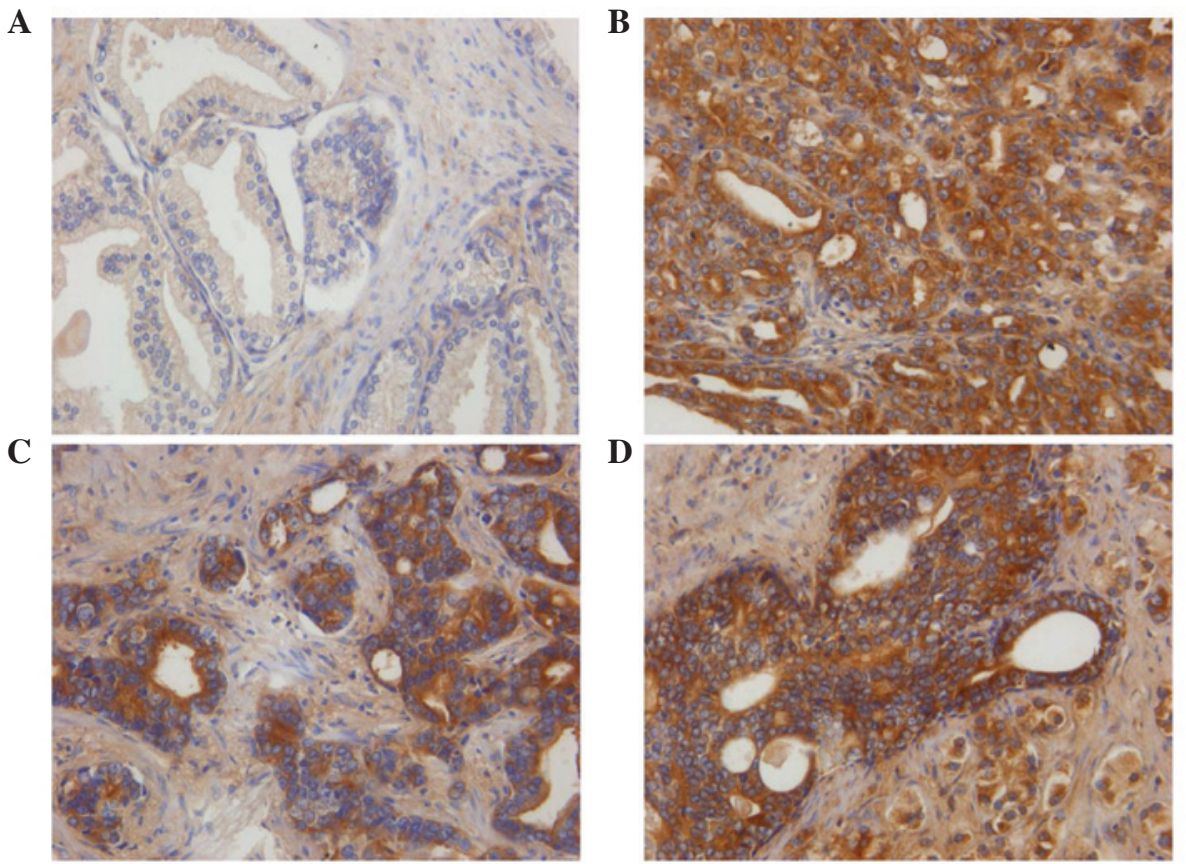

Figure 1. Immunostaining of ILK protein in BPH and PCa specimens. (A) Negative expression of ILK in BPH; (B-D) positive expression of ILK in PCa tissues. Magnification, x200. ILK, integrin-linked kinase; BPH, benign prostatic hyperplasia; PCa, prostate cancer.

decreased in DU145 cells compared with the control cells (Fig. 2). To assess the effects of ILK silencing on the growth of DU145 cells, cell viability was assessed using the MTT assay. ILK silencing was found to have significant inhibitory effects on the proliferation of ILK shRNA-transfected cells compared with the empty vector-transfected cells (Fig. 3). Additionally, wound healing assay was performed in the two groups of cells. ILK-depleted cells spread along the wound edges were characterized by slower migration compared with the vector-transfected cells at 12 or $24 \mathrm{~h}$, indicating that inhibition of ILK suppressed tumor cell migration (Fig. 4). Moreover, ILK-depleted cells and control cells were doublestained with Annexin V and PI, followed by flow cytometric analysis. An increased apoptosis rate was observed in ILK shRNA-transfected DU145 cells compared with the control cells, following RNA interference treatment for $48 \mathrm{~h}$ (Fig. 5). Taken together, these findings provide evidence

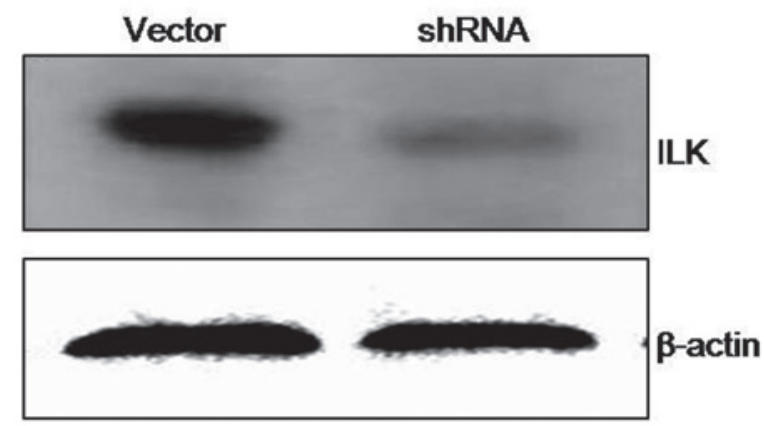

Figure 2. ILK shRNA inhibits the expression of ILK. DU145 cells were transiently transfected with shRNA targeting ILK. Western blot analysis was performed $48 \mathrm{~h}$ following transfection. ILK, integrin-linked kinase; shRNA, short hairpin RNA. 


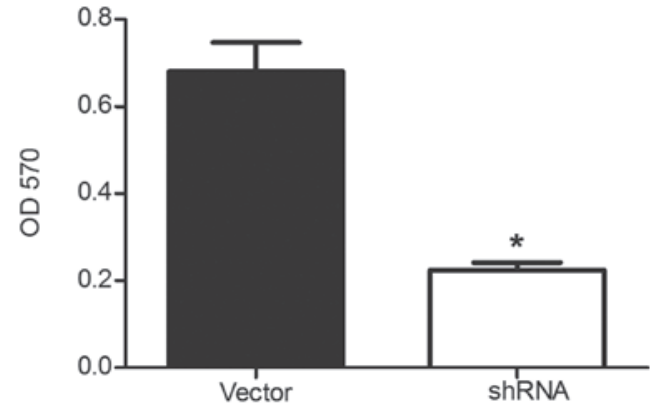

Figure 3. Silencing of integrin-linked kinase (ILK) inhibits the proliferation of DU145 cells. The relative growth optical density (OD) values of DU145 cells assessed by MTT are shown. The growth of cells transfected with ILK shRNA was significantly decreased compared with the growth of control cells, ${ }^{*} \mathrm{P}<0.05$. Data are expressed as the mean \pm SEM. shRNA, short hairpin RNA.

A

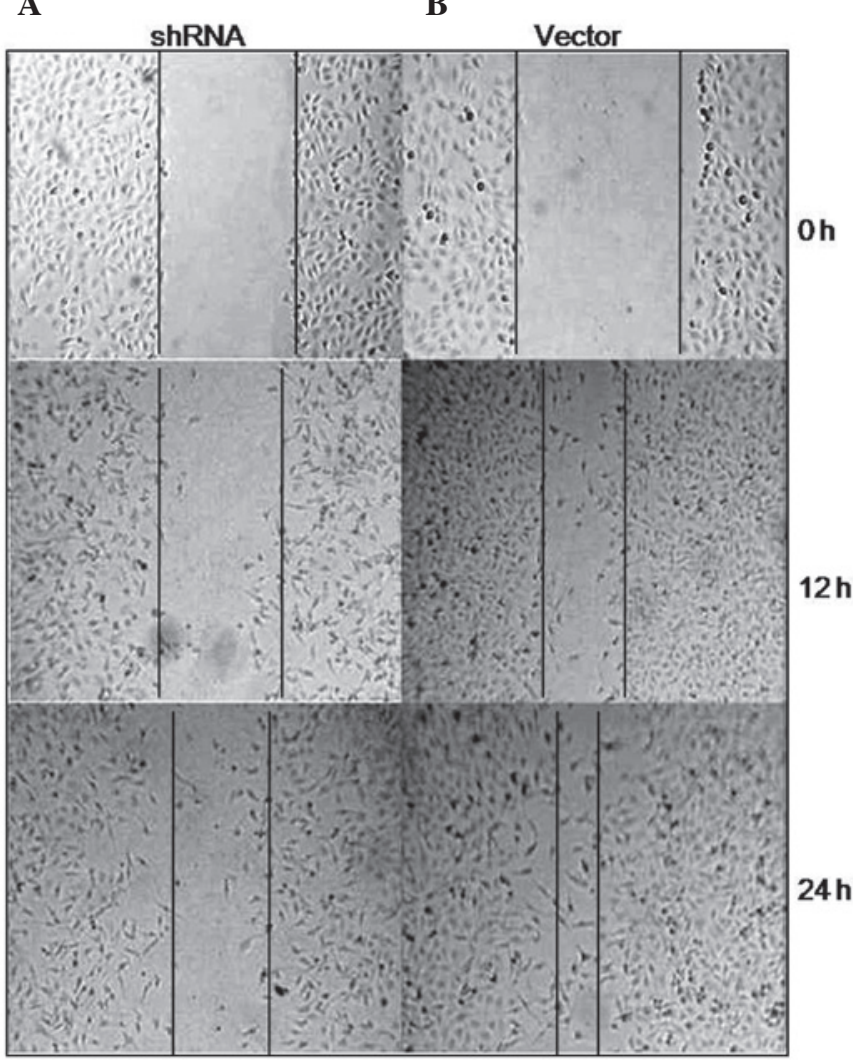

Figure 4. ILK knockdown slowed down cell migration as assessed by wound healing assay. Cells transfected with (A) ILK shRNA and (B) empty vector. Transfected cells were grown to confluence and a wound was created. The edge of the wound was monitored and cells were imaged at the indicated time points. ILK, integrin-linked kinase; shRNA, short hairpin RNA.

that ILK silencing attenuates migration, proliferation and survival of PCa cells.

Effect of ILK knockdown on the downstream Akt pathway. ILK is a multifunctional intracellular effector of cell-matrix interactions, which is involved in cancer cell growth and survival through modulation of downstream targets, notably Akt, which plays a role in determining cell behavior with regards to proliferation, migration and survival $(7,16)$. In order to determine whether Akt activity is decreased following
A

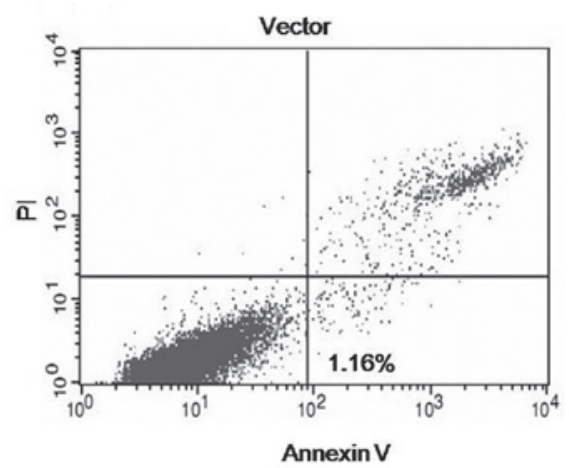

B

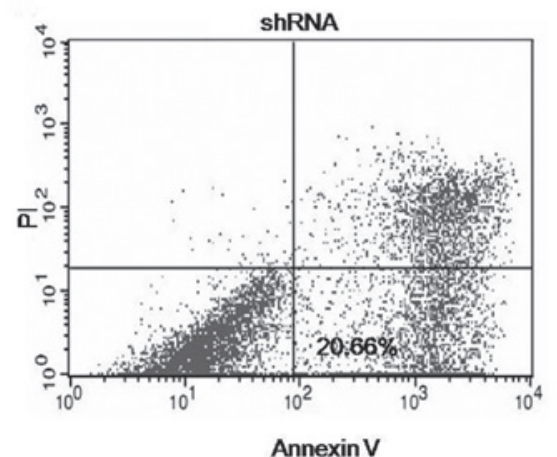

C

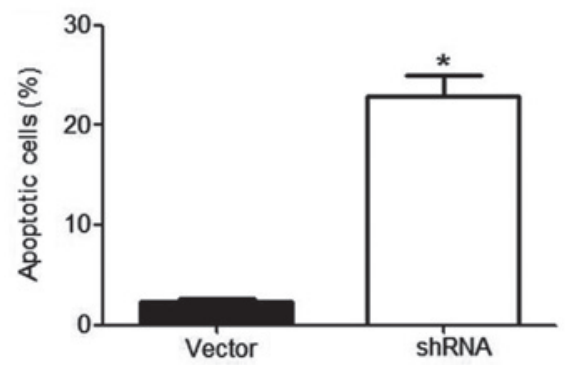

Figure 5. Early cell apoptosis was assessed by flow cytometry using Annexin V/PI double staining. Bottom left, living cells; top left, necrotic cells; bottom right, early apoptotic cells; top right, late apoptotic cells. (A) Control group of vector-transfected cells; (B) ILK shRNA-transfected cells. (C) ILK knockdown significantly induced PCa cell apoptosis, ${ }^{*} \mathrm{P}<0.05$. ILK, integrinlinked kinase; PCa, prostate cancer; shRNA, short hairpin RNA.

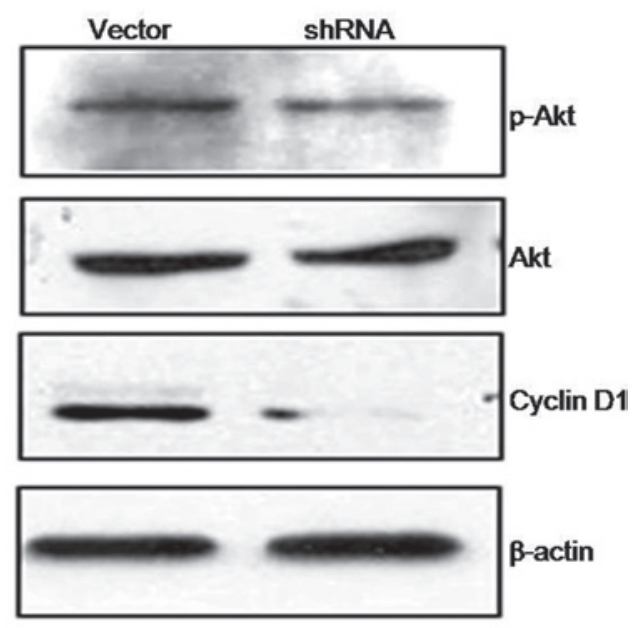

Figure 6. Role of ILK in Akt signaling. DU145 cells were transfected with control or ILK shRNA for $48 \mathrm{~h}$, followed by immunoblot analysis of Akt, phosphorylated Akt and cyclin D1. $\beta$-actin was used as an internal control. ILK, integrin-linked kinase; shRNA, short hairpin RNA. 
ILK suppression in PCa cells, the phosphorylated Akt and its downstream molecule were detected by western blot analysis. Phosphorylated Akt was found to be significantly decreased combined with the downregulation of cyclin D1, which is one of the most important proteins in tumor progression (Fig. 6).

Effect of ILK knockdown on PCa growth in vivo. The effects of ILK depletion on PCa xenograft growth were assessed in vivo. ILK shRNA or vector were transfected ex vivo into DU145 cells. The cells were subsequently implanted in the subcutaneous layer of immunodeficient mice $(n=10)$ and tumor sizes were measured after 4 weeks. ILK shRNA-transfected cells generated xenografts $\left(19.0 \pm 1.1 \mathrm{~mm}^{3}\right)$ that were significantly smaller compared with control vector-transfected xenografts (153.4 $\pm 8.0 \mathrm{~mm}^{3} ; \mathrm{P}<0.05$; Fig. 7).

\section{Discussion}

Interaction between cell-cell and cell-extracellular matrix, often triggered through the signals mediated by integrin and growth factor receptors, controls the growth, differentiation, migration and apoptosis of cells $(6,17,18)$. ILK is a cellular signaling protein with Ser/Thr protein kinase activity, and is involved in the formation of the signaling coupling complex of integrin and growth factor receptor (7). Due to its relevance to these receptors, ILK participates in the regulation of basic cell functions, such as the interaction between cell and extracellular matrix, and intracellular proliferation signaling. Thus, minor alterations in ILK expression or activity, could result in changes of the corresponding signaling pathway and the subsequent cell cycle, and may cause abnormal cell growth and proliferation, leading to tumor formation $(19,20)$.

ILK expression has been found to be increased in many types of human cancer (11). Previous studies have demonstrated that ILK expression is also positively correlated with tumor grade and inversely correlated with the 5-year patient survival rate (14). In the present study, $63 \mathrm{PCa}$ and $11 \mathrm{BHP}$ samples were subjected to immunohistochemical analysis to assess the levels of ILK in tumorous and BPH tissues. ILK was found to be overexpressed in $>40 \%$ of tumorous prostate tissues compared with BPH tissues. The positive rate of ILK expression in PCa tissues increased with the increasing Gleason score, although the difference was not statistically significant. These results suggest that ILK plays a role in the development and progression of PCa.

Despite the fact that the potential significance of ILK in prostatic carcinogenesis has been previously reported, the functional role of ILK in advanced PCa and the associated pathways have yet to be fully elucidated. It has been demonstrated that siRNA ILK and other small-molecule ILK inhibitors inhibit proliferation and induce apoptosis in breast cancer cells, melanoma and acute myeloid leukemia stem cells $(12,13,21)$. The effects of ILK knockdown on the growth, migration and apoptosis of PCa cells should be further investigated. In the present study, endogenous ILK expression was silenced in the PCa cell line DU145 using specific shRNA. The properties of the ILK-depleted cells were then analyzed and compared with those of control vector-transfected cells using various functional assays. ILK knockdown was found to suppress cell proliferation and to induce PCa cell apoptosis.
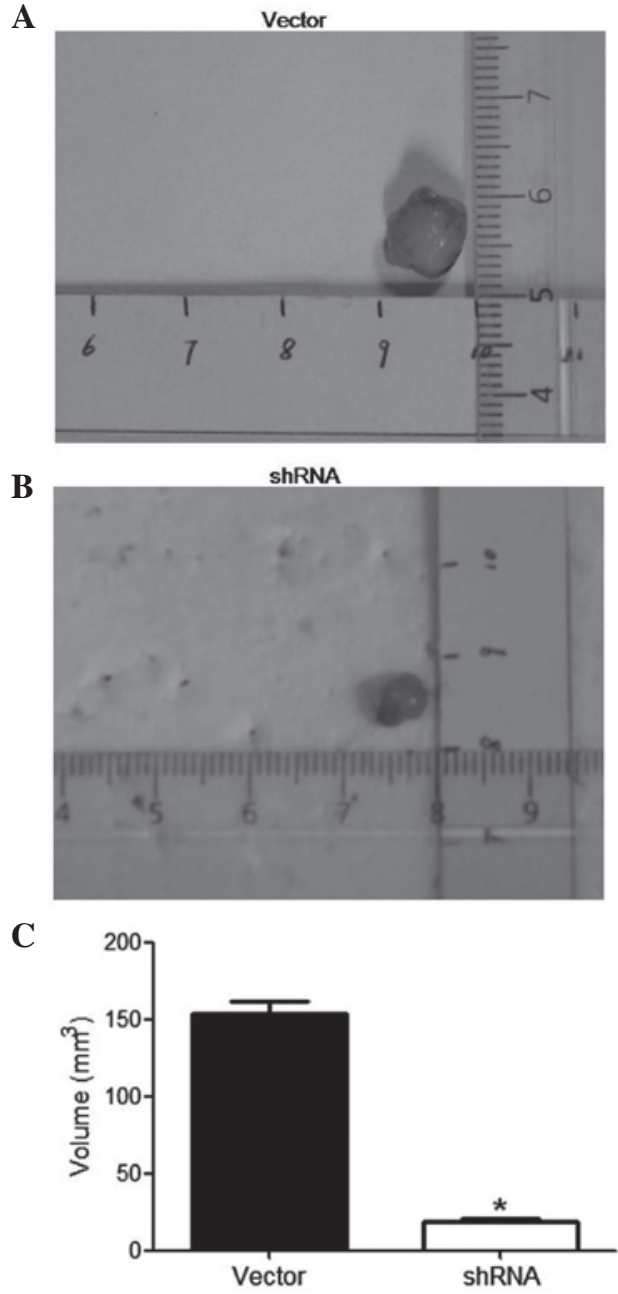

Figure 7. Downregulation of ILK expression reduces DU145 cell xenograft growth in vivo. (A) Control group; (B) ILK shRNA group; (C) the tumor volume of the ILK shRNA group was significantly reduced compared with the control group. "P $<0.05$. ILK, integrin-linked kinase; shRNA, short hairpin RNA.

In addition, cell motility was also impeded by ILK depletion. The present study provides the first evidence regarding the functional loss of ILK expression in vivo. PCa cells with suppressed ILK expression displayed a reduced ability to form tumors in nude mice. These observations provide direct evidence that ILK has a critical role in the initiation phase of androgen-insensitive prostate tumor induction.

The exact mechanism of ILK knockdown in inhibiting PCa cell growth and inducing apoptosis has yet to be elucidated. Akt, phosphorylation of which at the serine 473 position by ILK in a phosphatidylinositol 3-kinase (PI3K)-dependent manner, has been reported to be the main downstream player in ILK signaling pathway (19), while persistent activation of the Akt signaling cascade is prominent in various types of human cancer. Thus, depletion of ILK expression or activity could slow down the progression of several types of cancer, that may be involved in the Akt pathway. A previous study has shown that inhibition of ILK could also suppress the activation of Akt in PTEN-mutant prostate cancer cells (20). Activated Akt phosphorylates or regulates a number of cellular proteins that are implicated in tumorigenesis and motility (22). In the present study, we showed that blockade of the ILK pathway 
attenuated tyrosine phosphorylation of the Akt pathway and its downstream molecule cyclin D1, which significantly affected malignant features of tumor cells. Thus, ILK-Akt signaling is suggested to be an important regulatory pathway in advanced PCa. Further studies are needed to investigate the exact mechanisms of ILK involved in PCa.

In summary, the present study showed that ILK is overexpressed in human PCa. ILK silencing significantly attenuates PCa tumorigenicity as assessed by western blot analysis, proliferation assay, flow cytometry and wound healing assay in vitro, and using a xenograft model in vivo. ILK knockdown is suggested to inhibit the biological behavior of advanced $\mathrm{PCa}$ cells through attenuating Akt activity. The present preliminary study provides the first direct evidence regarding a potential association of ILK knockdown with antitumor progression. Although further investigation and validation is needed concerning the underlying mechanisms of ILK depletion affecting PCa progression, ILK is suggested to be a potential therapeutic target for $\mathrm{PCa}$.

\section{Acknowledgements}

This study was supported by the National Natural Science Foundation of China (81141029). The authors would like to thank the Department of Pathology of Peking University for kindly providing the DU145 human prostate cancer cell line and for the technical support in immunohistochemical analysis.

\section{References}

1. Jemal A, Siegel R, Xu J and Ward E: Cancer statistics, 2010. CA Cancer J Clin 60: 277-300, 2010.

2. Loblaw DA, Mendelson DS, Talcott JA, et al: American Society of Clinical Oncology recommendations for the initial hormonal management of androgen-sensitive metastatic, recurrent, or progressive prostate cancer. J Clin Oncol 22: 2927-2941, 2004.

3. Feldman BJ and Feldman D: The development of androgen-independent prostate cancer. Nat Rev Cancer 1: 34-45, 2001.

4. Hannigan GE, Bayani J, Weksberg R, et al: Mapping of the gene encoding the integrin-linked kinase, ILK, to human chromosome 11p15.5-p15.4. Genomics 42: 177-179, 1997.

5. Hannigan G, Troussard AA and Dedhar S: Integrin-linked kinase: a cancer therapeutic target unique among its ILK. Nat Rev Cancer 5: 51-63, 2005.

6. Gil D, Ciolczyk-Wierzbicka D, Dulinska-Litewka J, Zwawa K, McCubrey JA and Laidler P: The mechanism of contribution of integrin linked kinase (ILK) to epithelial-mesenchymal transition (EMT). Adv Enzyme Regul 51: 195-207, 2011.
7. Gao J, Zhu J, Li HY, Pan XY, Jiang R and Chen JX: Small interfering RNA targeting integrin-linked kinase inhibited the growth and induced apoptosis in human bladder cancer cells. Int J Biochem Cell Biol 43: 1294-1304, 2011.

8. Ahmed N, Riley C, Oliva K, Stutt E, Rice GE and Quinn MA: Integrin-linked kinase expression increases with ovarian tumour grade and is sustained by peritoneal tumour fluid. J Pathol 201: 229-237, 2003.

9. Bravou V, Klironomos G, Papadaki E, Taraviras S and Varakis J: ILK over-expression in human colon cancer progression correlates with activation of beta-catenin, down-regulation of E-cadherin and activation of the Akt-FKHR pathway. J Pathol 208: 91-99, 2006.

10. Intaraprasong P, Assi K, Owen DA, et al: Expression of integrin-linked kinase is not a useful prognostic marker in resected hepatocellular cancer. Anticancer Res 27: 4371-4376, 2007.

11. Goulioumis AK, Bravou V, Varakis J, Goumas P and Papadaki H: Integrin-linked kinase cytoplasmic and nuclear expression in laryngeal carcinomas. Virchows Arch 453: 511-519, 2008.

12. Muranyi AL, Dedhar S and Hogge DE: Targeting integrin linked kinase and FMS-like tyrosine kinase-3 is cytotoxic to acute myeloid leukemia stem cells but spares normal progenitors. Leuk Res 34: 1358-1365, 2010.

13. Wong RP, Ng P, Dedhar S and Li G: The role of integrin-linked kinase in melanoma cell migration, invasion, and tumor growth. Mol Cancer Ther 6: 1692-1700, 2007.

14. Graff JR, Deddens JA, Konicek BW, et al: Integrin-linked kinase expression increases with prostate tumor grade. Clin Cancer Res 7: 1987-1991, 2001.

15. Sasaki T, Ryo A, Uemura H, et al: An immunohistochemical scoring system of prolyl isomerase Pin1 for predicting relapse of prostate carcinoma after radical prostatectomy. Pathol Res Pract 202: 357-364, 2006

16. Hou Y, Mortimer L and Chadee K: Entamoeba histolytica cysteine proteinase 5 binds integrin on colonic cells and stimulates NFkappaB-mediated pro-inflammatory responses. J Biol Chem 285: 35497-35504, 2010.

17. Buchheit CL, Rayavarapu RR and Schafer ZT: The regulation of cancer cell death and metabolism by extracellular matrix attachment. Semin Cell Dev Biol 23: 402-411, 2012.

18. Lu P, Takai K, Weaver VM and Werb Z: Extracellular matrix degradation and remodeling in development and disease. Cold Spring Harb Perspect Biol 3: a005058, 2011.

19. Delcommenne M, Tan C, Gray V, Rue L, Woodgett J and Dedhar S: Phosphoinositide-3-OH kinase-dependent regulation of glycogen synthase kinase 3 and protein kinase B/AKT by the integrin-linked kinase. Proc Natl Acad Sci USA 95: 11211-11216, 1998.

20. Persad S, Attwell S, Gray V, et al: Inhibition of integrin-linked kinase (ILK) suppresses activation of protein kinase B/Akt and induces cell cycle arrest and apoptosis of PTEN-mutant prostate cancer cells. Proc Natl Acad Sci USA 97: 3207-3212, 2000.

21. Troussard AA, McDonald PC, Wederell ED, et al: Preferential dependence of breast cancer cells versus normal cells on integrin-linked kinase for protein kinase B/Akt activation and cell survival. Cancer Res 66: 393-403, 2006.

22. Hers I, Vincent EE and Tavare JM: Akt signalling in health and disease. Cell Signal 23: 1515-1527, 2011. 